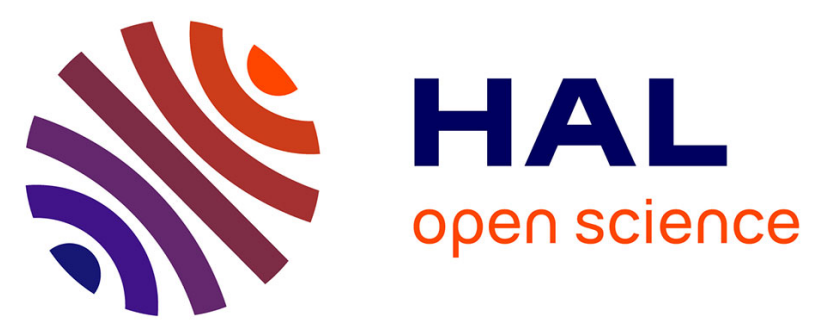

\title{
Control of a hybrid robotic system for computer-assisted interventions in dynamic environments
}

\author{
Gabrijel Smoljkic, Gianni Borghesan, Alain Devreker, Emmanuel Vander \\ Poorten, Benoît Rosa, Herbert de Praetere, Joris de Schutter, Dominiek \\ Reynaerts, Jos Vander Sloten
}

\section{To cite this version:}

Gabrijel Smoljkic, Gianni Borghesan, Alain Devreker, Emmanuel Vander Poorten, Benoît Rosa, et al. Control of a hybrid robotic system for computer-assisted interventions in dynamic environments. International Journal of Computer Assisted Radiology and Surgery, 2016, 11 (7), pp.1371 - 1383. 10.1007/s11548-015-1333-8 . hal-01765107

\section{HAL Id: hal-01765107 \\ https://hal.science/hal-01765107}

Submitted on 12 Apr 2018

HAL is a multi-disciplinary open access archive for the deposit and dissemination of scientific research documents, whether they are published or not. The documents may come from teaching and research institutions in France or abroad, or from public or private research centers.
L'archive ouverte pluridisciplinaire HAL, est destinée au dépôt et à la diffusion de documents scientifiques de niveau recherche, publiés ou non, émanant des établissements d'enseignement et de recherche français ou étrangers, des laboratoires publics ou privés. 


\title{
Control of a hybrid robotic system for computer-assisted interventions in dynamic environments
}

\author{
Gabrijel Smoljkic · Gianni Borghesan · Alain Devreker • Emmanuel Vander \\ Poorten - Benoit Rosa · Herbert De Praetere · Joris De Schutter · Dominiek \\ Reynaerts · Jos Vander Sloten
}

Received: date / Accepted: date

\begin{abstract}
Purpose Minimally invasive surgery is becoming the standard treatment of care for a variety of procedures. Surgeons need to display a high level of proficiency to overcome the challenges imposed by the minimal access. Especially when operating on a dynamic organ, it becomes very difficult to align instruments reliably and precisely. In this paper, a hybrid robotic system and a dedicated robotic control approach are proposed to assist the surgeon performing complex surgical gestures in a dynamic environment.

Methods The proposed hybrid robotic system consists of a rigid robot arm on top of which a continuum robot is mounted in series. The continuum robot is locally actuated with McKibben muscles. A control scheme is adopted based on quadratic programming framework. It is shown that this framework allows enforcing a set of constraints on the pose of the tip, as well as of the instrument shaft, which is commanded to slide in and out through the entry point.

Results Through simulation and experiments it is shown how the robot tool-tip is able to follow sinusoidal trajectories of $0.37 \mathrm{~Hz}$ and $2 \mathrm{~Hz}$, corresponding to motion due to breathing and heartbeat respectively, while maintaining the instrument shaft pivoting nicely about the entry point. The positioning and tracking accuracy of such system is shown to lie below $3 \mathrm{~mm}$ in position and $5^{\circ}$ in angle.
\end{abstract}

Herbert De Praetere is with

UZ Leuven, Cardiac surgery,

Herestraat 49, 3000 Leuven, Belgium

E-mail: herbert.depraetere@uzleuven.be

All other authors are with

KU Leuven, Department of Mechanical Engineering,

Celestijnenlaan 300C, B-3001 Leuven, Belgium

E-mail: name.surname@kuleuven.be
Conclusion The results suggest a good potential for applying the proposed technology to assist the surgeon during complex robot-assisted interventions. It is also illustrated that even when using flexible hence relatively safe end-effectors, it is possible to reach acceptable tracking behaviour at relatively high frequencies.

Keywords Constraint based control - Continuum robot - Motion compensation - Heart surgery . Transapical valve implantation

\section{Introduction}

Minimally invasive surgery (MIS) is becoming the standard treatment for many surgical procedures. Shorter patient recovery time, reduced trauma, reduced inflammatory reaction and potentially lower medical costs are some of the claimed benefits. For example in the case of heart surgery the procedure could be performed on a beating heart as opposed to open heart surgery, which more frequently needs an arrested heart and a cardiopulmonary bypass. If operated on an arrested heart, the heart is assessed in a non-physiological condition. In the beating heart approach, the surgeon can more easily evaluate the outcome of her/his actions and adjust the intervention on the spot to optimize the clinical outcome. Whereas the patient is seeing multiple and clear benefits, this stands in contrast to a significantly increased difficulty in execution of the procedure itself, due to reduced access and reduced dexterity. The success of the procedure is therefore determined by the ability of the surgeon to overcome these technical challenges and her/his ability to correctly handle and align surgical instruments, and manipulate tissue or anatomic structures. To simplify the problem, nowadays, one often resorts to rapid pacing, minimizing cardiac 
motion and therefore simplifying instrument control. Rapid pacing is an established technique for temporary heart arrest. For example in the procedures such as Transcatheter Aortic Valve replacement (TAVI) [5], rapid pacing is used when the arrest of left ventricular ejection is required. The periods where rapid pacing is used are however to be kept minimal in order to ensure hemodynamic stability. This is especially critical in patients with depressed left ventricular function and/or non-revascularized coronary artery disease [16].

Above mentioned limitations could potentially be overcome by introducing a dynamic robotic system that guides the positioning and deployment of surgical instruments in the moving environment. For example in the work by Kesner [14], a motion compensation control strategy for a single Degree of Freedom (DoF) robotic catheter was demonstrated. This study focused on solving the problems of catheter backlash and friction resulting from insertion of the catheter inside the guiding rigid sheath. The kinematics of such system is straightforward since the resulting insertion and retraction of the catheter results in a collocated motion of the robotic catheter tip w.r.t. the guiding sheath. The access port for surgical instruments resulting from the MIS approach particularly limits the dexterity of the surgical instruments, hence additional degrees of freedom are required to correctly position and align the instruments. By introducing the instrument through the access port(trocar), the surgeon is effectively losing two degrees of freedom. Her/his motions are restricted to insertion/retraction, rotation and pivoting about the entry point. Moreover, the pivot point creates a fulcrum effect that inverts the movements of the tip of the instrument. By using an instrument which offers two additional local DoFs past the trocar point, instrument dexterity can be restored, allowing for complete control over position and orientation of the tool. For example, a possible compliant 2-DoF surgical instrument was presented in [21]. Such compliant structure, nowadays often referred to as continuum robot $[22,27]$, would provide additional local DoFs that can overcome the motion constraints that are imposed by the access port. The combination of a rigid robot with a tip-mounted active continuum robot proposed in this work would restore the 6 DoFs required for correctly positioning and alignment of the surgical instruments. Fig. 1 illustrates conceptually how such a system could be used for example for a robot-assisted transapical, transcatheter aortic valve implantation. The prosthetic valve would be mounted here on top of the flexible surgical instrument. In more challenging procedures such as mitral valve repair and mitral valve replacement, such robotic approaches could bring even more bene-

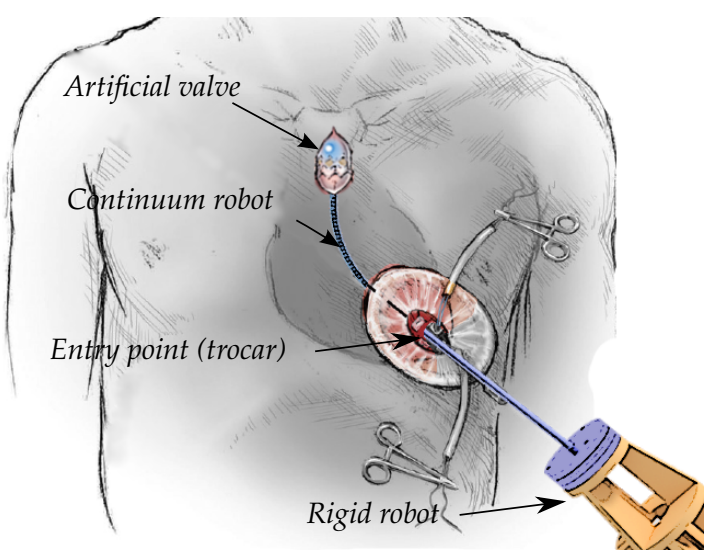

Fig. 1 Illustration of an exemplary robot-assisted heart procedure. In trans-catheter transapical aortic valve implantation the native valve has to be replaced by a prosthetic valve which could be mounted on a dynamically controlled hybrid robotic system as illustrated here.

fits. The use of such hybrid-robotic mechanism for operation on a beating heart was also demonstrated in the work of Thienprapa [24]. The system was used to position the instrument tip on a location in the heart where a possible foreign body could be captured. Even though operating on a beating heart, the control strategy does not involve active heart beating compensation techniques and the overall system, as claimed by the authors, features slow motion. In this paper we propose a novel hybrid robotic system, where a pneumatically actuated continuum robot featuring 2-DoF was used to follow a relatively fast heart beating motion.

Continuum robots are considered more and more for use in surgical interventions. Their ability to bend and flex upon contact with the environment is considered extremely appealing especially from a safety point of view. When compared to their rigid counterparts such as the Intuitive Surgical Da Vinci system [12], the inherent compliance many of the continuum robots exhibit makes it easier to keep force levels down. Nevertheless, the control of continuum robots is considerably more involved since their kinematic behaviour is largely dependant on various forces acting on the robot (such as environment interaction forces or gravity). In recent years, this problem attracted a lot of attention by the research community. A variety of models as well as different designs of pneumatically driven continuum robots have been proposed. To name a few, the deflection of the pneumatically driven robots under the presence of external loads was treated in [26]. Here a Cosserat rod model of the robot was used to account for deflections resulting from the gravitational loads. Another example includes modelling of a multisegment robot through a lumped mass-spring-damper model [13]. The behaviour of pneumatically driven ro- 
bots with variable lengths was described in [10]. This work targeted in particular the kinematics of robots which experience contraction and elongation during their actuation. Another concept of a pneumatically driven robot used as an active colonoscope was demonstrated in [4]. Here a constant curvature assumption was used to model the continuum robot kinematics. Whereas these aforementioned designs are not meant to be used as surgical tools for heart surgery mainly due to their large size, the main concepts of pneumatic actuation and kinematic modelling are also valid for the case of the continuum robot treated in this paper. Since the goal of this paper is to evaluate the ability of the robotic system to follow complex trajectories under a complex set of constraints, we will deal mainly with free space motion. A simpler constant curvature continuum robot model can therefore be used here [28].

Multiple and complex motion constraints follow directly from the nature of the envisioned transapical MIS procedure. Since these constraints need to be expressed in different, time-varying coordinate frames, this largely complicates the formulation of appropriate robot controllers. Here, the task-frame formalism by Mason [19] forms an elegant solution allowing easy expression of the different control objectives and according derivation of joint level controllers. The Task Frame Formalism is the ancestor of many frameworks including $i$ ) the whole body framework (WBF, [15]) a torque-resolved, priority-based scheme ii) the Stack of Task (SoT, [18]), with great emphasis on optimization of the solution computation, and iii) the instantaneous task specification using constraints (iTaSC, [7]) and one of the later developments eTC(expressiongraph-based Task Controller, [1]) which, taking advantage of alternative spatial representations, enriches the flexibility of task description.

In our work the eTC framework is adopted to control a hybrid robotic system composed of rigid and continuum elements. The approach proposed in this work is general in the sense that it does not discriminate between flexible or rigid parts of the system as long as they are modelled in an adequate manner. Both the controller and the actual system are validated in a scenario where following fast trajectories is required. Here, position tracking of the instrument tip is shown to be possible while maintaining a rotational center-ofmotion.

The paper is built up as follows. crefsec:hybridsystem gives an overview of the system and of the control framework (Sec. 3) that is being used. The so-called surgical scene which summarizes the collection of objects and coordinate frames of interest, along with imposed constraints is introduced in Sec. 4. Experimental

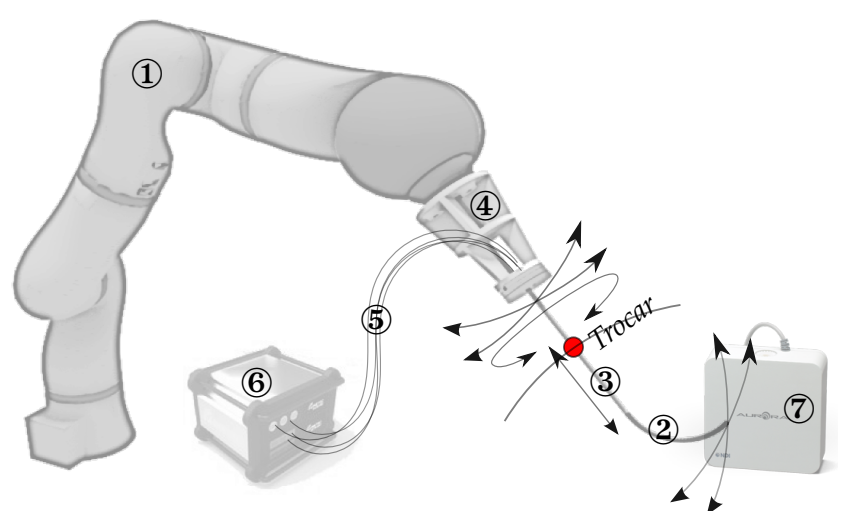

Fig. 2 Hybrid robotic system consisting of a flexible continuum robot mounted on a rigid robot arm. The rigid robot provides 6 DoFs to the tip, of which 2 are sacrificed to comply with the kinematic constraint at the entry point into the body. Two additional DoFs are offered by the continuum robot tip which restore the instrument dexterity in the presence of the trocar constraint.

results are reported in Sec. 5.3, and finally conclusions are drawn in Sec. 6.

\section{System overview}

Fig. 2 shows the proposed hybrid-robotic system, where the arrows indicate the possible motions the robot is able to perform. Similar to manual operation, the rigid part of the robot can also only execute: tool insertion through the entry point, rotation of the tool about the tool axis and tool pivoting around the entry point. In addition, the tool tip can be bent in 2 directions. The hybrid robot employed in this work consists of a pneumatically actuated continuum robot (2), mounted on a longer shaft (3) which is used to pass the surgical tool through the trocar(indicated by the red circle). This shaft is rigidly connected to a 3D-printed mounting stage (4) which is finally mounted on the rigid robot (1). The type of the rigid robot employed in this study is a KUKA LWR robot (KUKA, Germany) featuring 7 DoF. The continuum robot control box (6) contains valves to supply the pressure to the continuum robot through the long pressure tubes (5). An electromagnetic (em) field generator module (NDI, Aurora) (7) is placed in the vicinity of the site of interest, giving absolute position measurements (with respect to the coordinate frame of the field generator) of the continuum robot.

The remainder of this section describes the continuum robot design (Sec. 2.1) and characterizes its kinematic properties (??). 


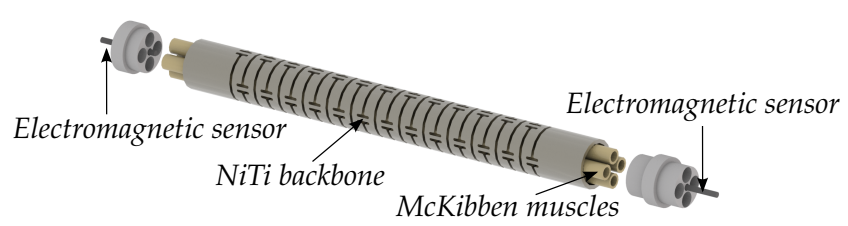

Fig. 3 The robot is built from a NiTi tube designed to achieve the desired bending. Four McKibben muscles are embedded inside the NiTi tube. At the connection pieces at the tip and the rear two electromagnetic tracking sensors are attached.

\subsection{Continuum robot - Design and Actuation}

The continuum robot, depicted in Fig. 3, is a composite structure actuated with four McKibben muscles (a type of pneumatic artificial muscles, PAM) embedded into a flexible nitinol (NiTi) tube. In order to achieve the desired maximal bending and stiffness, the tube has been cut using wire-EDM, as shown in Fig. 3. The resulting structure is $66 \mathrm{~mm}$ long and has an external diameter of $7 \mathrm{~mm}$. Each muscle is $66 \mathrm{~mm}$ long, has an initial diameter of $2.1 \mathrm{~mm}$, and it is fastened at each side of the continuum robot.

The actuation is realised pneumatically. An increase of pressure induces a radial expansion of the muscle, as well as an axial contraction, and thereby an axial pulling force. This pulling force is applied to the structure, making it bend. Characterized by a high power density, McKibben muscles are also inherently more compliant than other actuation principles. An extensive characterization of artificial muscles can be found in the literature $[6,11,25]$. Note that the surrounding NiTi tube acts as a return spring, so that the continuum robot straightens back if the pressure is released. The four muscles are symmetrically distributed within the NiTi tube, so that bending motions in two directions are achieved by pressurizing one or more artificial muscles.

The bending curvature of the structure is defined by the composite stiffness of both the muscles and of the NiTi tube, as well as the pressure to force ratio of the artificial muscles. The relation between the curvature of the continuum robot and the applied pressure can then be expressed with a parameter $K$ which has a unit of $[1 / \mathrm{Pa} \cdot \mathrm{mm}]$. This parameter was obtained experimentally by linearly increasing the pressure in one of the muscles and measuring the bending angle using the embedded $e m$ sensors. The obtained value for this parameter is $K=0.01431 / \mathrm{Pa} \cdot \mathrm{mm}$. The pressure range in each of the muscles goes from $0.5 b a r$ to $7 b a r$. This minimal pressure in each of the muscles ensures a proper pre-pressurisation and moves the working range of the muscle away from the initial "backlash" of the muscle which arises around zero pressure [9].

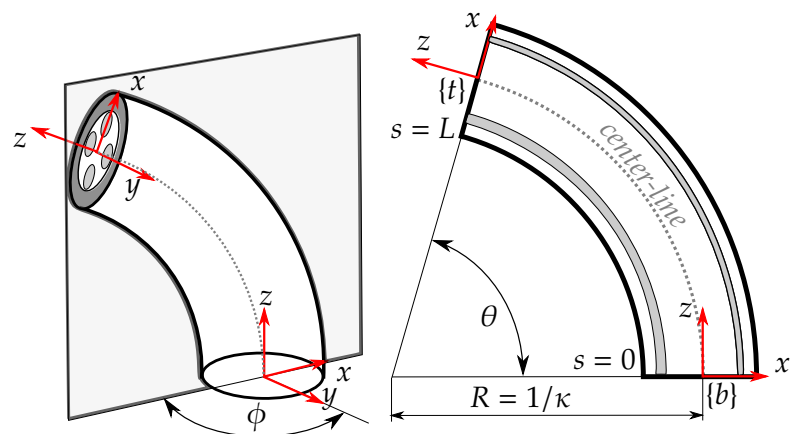

Fig. 4 The robot independent mapping gives the description of the robot shape w.r.t. the arc parameters: $\kappa$ and $\phi$.

Two em tracking sensors are embedded at both extremities of the continuum robot. These $\mathrm{em}$-sensors allow measurement of the relative pose of the tip of the continuum robot with respect to its base.

\subsection{Continuum robot - Kinematics and Differential Kinematics}

In the present work we employ a constant curvature model to model the continuum robot. Following the guidelines outlined in [28], the kinematics of the continuum robot can be divided into two parts. The robot independent mapping describes the shape of the continuum robot as a function of the arc parameters: the curvature $\kappa$ and the angle of rotation $\phi$. From the robot independent mapping, a link can be made towards the robot actuation space $q_{c}$. Since the 4 McKibben muscles are aligned with the principal axis of the continuum robot, unequal pressurization of the different muscles causes the flexible robot to bend in a plane defined with this principal axis and the axis tangent to the centreline (z-axis). Given that the muscles can only work in an antagonistic way (i.e. pull-pull configuration), 2 muscles are required to span both sides of the bending plane. The control variables $q_{c}$ of the continuum robot can be thus defined as:

$q_{1}=p_{1}-p_{3}$

$q_{2}=p_{2}-p_{4}$

where $q_{1}$ and $q_{2}$ represent the control space variables and $p_{j}, j \in[1, \ldots, 4]$ is the pressure in each of the muscles. The equations ?? are not well defined and they allow an infinite number of combinations for pressures $\left(p_{1} p_{3}\right)$ and $\left(p_{2} p_{4}\right)$. Additional constraints can be imposed here based on the requirement of prepressurisation at $0.5 b a r$ and minimal overall pressure in the system.

A further relation between the continuum robot control space $q_{c}=\left[q_{1}, q_{2}\right]$ and the variables of the robot 
independent mapping $q_{i}=[\kappa, \phi]$ can be written as:

$q_{1}=K \kappa \cos \phi$,

$q_{2}=K \kappa \sin \phi$,

where $K$ is the muscle coefficient which relates the pressure to curvature.

From [28], the forward kinematics transformation matrix for the robot independent mapping can be written as:

${ }_{b} \boldsymbol{T}^{t}(\kappa, \phi)=\left[\begin{array}{cccc}\cos \phi \cos \kappa L & -\sin \phi & \cos \phi \sin \kappa L & \frac{\cos \phi(1-\cos \kappa L)}{\sin (1-\cos \kappa L)} \\ \sin \phi \cos \kappa L & \cos \phi & \sin \phi \sin \kappa L & \frac{\sin \kappa^{\kappa} \kappa}{\frac{\operatorname{con} \kappa L}{\kappa}} \\ -\sin \kappa L & 0 & \cos \kappa L & 1 \\ 0 & 0 & 0 & 1\end{array}\right]$

Here $T$ is the homogeneous transformation matrix between the base frame $\{b\}$ of the continuum robot, and its tip frame $\{t\}$. The differential kinematics, and relative Spatial Manipulator Jacobian [20], of the continuum robot follows from (??):

${ }_{b} \mathbf{t}^{t}={ }_{b} \boldsymbol{J}^{t}\left[\begin{array}{c}\dot{\kappa} \\ \dot{\phi}\end{array}\right], \quad{ }_{b} \boldsymbol{J}^{t}=\left[\begin{array}{cc}\frac{\cos \phi(\cos \kappa L-1)}{\kappa^{2}} & 0 \\ \frac{\sin \phi(\cos \kappa L-1)}{\kappa^{2}} & 0 \\ \frac{-(\sin \kappa L-\kappa L)}{\kappa^{2}} & 0 \\ -L \sin \phi & 0 \\ L \cos \phi & 0 \\ 0 & 1\end{array}\right]$,

where the Jacobian matrix ${ }_{b} \boldsymbol{J}^{t}$ provides the mapping between the twist ${ }_{b} \mathbf{t}^{t}$ of the frame $\{t\}$ with respect to the base frame $\{b\}$, expressed in frame $\{b\}$, and the rate of change of robot independent variables $[\dot{\kappa} \dot{\phi}]^{T}$.

\section{Control framework}

The control of the robot is based on the eTC control framework, documented in [1]. This type of constraintbased control allows an easy definition and evaluation of tasks defined in different coordinate spaces and in different reference frames. The eTC framework is a velocity resolved control framework, and thus the desired quantities and control laws are expressed in terms of desired velocities. Each constraint defines one or more directions of the output space. In this space, at each time step, the desired velocity vector (i.e. the output rate-ofchange that we would like to observe during next time step) is computed as:

$\dot{y}_{d}^{\circ}=K_{p}\left(y_{d}-y\right)+\dot{y}_{d}$, where $y_{d}$ and $\dot{y}_{d}$ are the desired generalised positions and velocities, $y$ is the vector of position measurements, and $\dot{y}_{d}^{\circ}$ are the calculated(desired) velocities in the output space. $K_{p}$ is a (diagonal) matrix that contains the gains of the controller; its dimensions being $[1 / s]$. The gains here define the response time for each component of the constraint. Once the desired commanded velocities are determined, the problem of obtaining the control joint velocities is formulated as an optimization problem (see [8]):

$$
\begin{gathered}
\dot{\boldsymbol{q}}=\arg \min \left\|\dot{\boldsymbol{q}}^{T} \boldsymbol{H} \dot{\boldsymbol{q}}\right\|, \\
\text { subject to: } \quad \dot{\boldsymbol{q}} \in \arg \min \left\|A \boldsymbol{q}-\dot{y}_{d}^{\circ}+B \dot{\chi}_{u}\right\|_{W^{\prime}}
\end{gathered}
$$

where $W$ is a diagonal weighting matrix that, in case of conflicting constraints, determines which constraint will be dominant. $H$ is a matrix of joint weights that in case of redundancy will determine the inclination of a joint to move with respect to others. The $A$ matrix is the Jacobian matrix which is a combination of the rigid robot Jacobian ${ }_{w} \boldsymbol{J}^{b}$, the flexible robot Jacobian ${ }_{b} \boldsymbol{J}^{t}$, and the Jacobian mapping the Cartesian space to the output space where all constraints have been defined. The term $\boldsymbol{B} \dot{\chi}_{u}$ is a correction term (introduced in [7]) that is to be applied to the output, and that originates from movements of uncontrollable frames. In the case at hand this will include the (estimated) velocities of the beating heart [3]. The matrix $A$ and the term $B \dot{\chi}_{u}$ are computed automatically by the expressiongraph library. A detailed discussion on these derivations falls outside the scope of this work.

The weighting matrices $\boldsymbol{H}$ and $\boldsymbol{W}$ are also used to normalize joint variables and outputs that might have different physical dimensions. This is important for the proposed hybrid system as the joint variables of the continuum robot are expressed as curvature $\kappa$ (physical dimension of $[1 / \mathrm{mm}]$ ) and rotation angle $\phi$ (dimension [rad]). The same reasoning holds for matrix $W$ that combines linear and rotational constraints.

In the case at hand, the optimization scheme (2) computes the vector $\dot{\boldsymbol{q}}$ of generalised joint velocities, which includes: $i$ ) the joint velocities to be commanded to the rigid robot $[\mathrm{rad} / \mathrm{s}]$, ii) the commanded curvature variation $\dot{\kappa}[1 / \mathrm{mms}]$ of the flexible robot and iii) the commanded angle of rotation velocities $\dot{\phi},[\mathrm{rad} / \mathrm{s}]$, for rotation. The calculated velocities are given as a set-point for the rigid robot. In the case of the continuum robot, these values are integrated to obtain the targeted curvature $\kappa$ and rotation angle $\phi$. Latter are then converted into adequate pressure levels according to ????. 


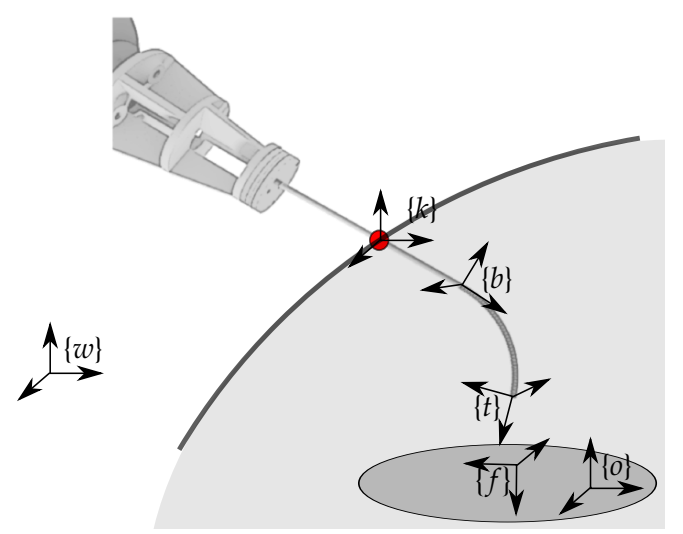

Fig. 5 Representation of the scene and the frames which define the relation between the objects in the scene.

\begin{tabular}{c||c|c|c|c}
\hline name & expressed in: & applied to: & subspaces & DoF \\
\hline tip pose & $\{0\}$ & $\{t\}$ & positions & 3 \\
trocar & $\{b\}$ & $\{k\}$ & porientation & 3 \\
& positions & 2 \\
\hline
\end{tabular}

Table 1 Constraints used in the experiment. Tip pose constraints are expressed in object frame $\{0\}$ while being applied in the tip frame $\{t\}$. The entry point constraint is expressed in $\{b\}$, but is applied to the entry point where the frame $\{k\}$ is defined.

\section{Scene and Constraints Description}

In constraint-based approaches, the definition of tasks is achieved in two steps. First frames are defined as they are attached to the robot(s) and object(s) of interest, then the outputs $y$ that are to be constrained are defined. Here, the objects of interest are the entry point (frame $\{k\}$ ) and the beating heart (frame $\{f\}$ ) at the point inside the patient's body where the treatment has to be delivered. Frame $\{0\}$ indicates the stationary, reference position of the heart. Frame $\{f\}$ represents in fact the uncontrollable frame resulting from the uncertainty related to the motion of the heart itself w.r.t. the frame $\{o\}$. On the robot, the frames $\{w\},\{b\}$, and $\{t\}$ are defined. The rigid part of the robot is located between frame $\{w\}$ and frame $\{b\}$. Latter is attached to the base of the flexible robot. Frame $\{t\}$ is conveniently attached to the tip of the continuum robot and indicates the frame of the surgical tool. Fig. 4 depicts the hybrid robotic system and the corresponding objects of interest.

In addition a number of constraints follow from the envisioned surgical task: i) The position and orientation of the tip frame relative w.r.t. the treatment site is to be controlled. These form a total of six constraints. ii) The instrument shaft is to be controlled along the $z$ axis of frame $\{b\}$ at the the entry point. This counts for two additional constraints. Tab.1 summarizes the list of constraints and the frames in which the constraints are defined.

The first set of constraints, labelled as tip pose, implements a position Cartesian control. This set of constraints ensure that the desired pose, i.e. zero relative orientation and positions, is expressed in a moving frame. If this frame is moving the velocity of motion will be compensated for.

The second set of constraints, labelled as trocar constraints, imposes that the shaft of the instrument, which is collinear to the $z$-axis of frame $\{b\}$, passes through the entry point. This behaviour is achieved by simply representing the entry point position $\mathbf{k}$ in the frame $\{b\}$,

${ }_{b} \mathbf{k}=\left({ }_{w} \boldsymbol{T}^{b}\right)^{-1}{ }_{w} \mathbf{k}$,

selecting the $x$ - and $y$-coordinates as part of the output vector,

$\boldsymbol{y}_{t r}=\left[\begin{array}{lll}1 & 0 & 0 \\ 0 & 1 & 0\end{array}\right]{ }_{b} \mathbf{k}$,

and imposing convergence to zero by means of (1) with null desired output vector $y_{d}$ and output desired velocity $\dot{y}_{d}$.

\section{Simulation and Experiments}

This section presents simulation and experimental results that were obtained in order to assess the performance of the proposed hybrid robotic system and the proposed control approach. The envisioned scenario follows from the case of transapical trans-catheter valve implantation on a beating heart. Note that at this point such scenario is a bit artificial as in reality the valve will be flushed away by the blood flow upon deployment. So a minimal arrest of the heart during the short period of valve deployment might be inevitable. Overall, the procedure requires the system to be able to follow motion due to heartbeat, but should at the same time respect the constraint of entry at the apex through the incision.

Fig.5 shows the experimental set-up. For sake of illustration of the principle working space of the hybrid robotic system, the tip of the robot was moved in several positions, while maintaining the orientation of the tip itself and of course maintaining the trocar constraint. Methods such as described in [23] can be used to estimate the location of an optimal pivot point. Here, the position of the tip frame $\{t\}$ and the frame $\{b\}$ are both measured by the em tracking sensors. By expressing the position of the $\mathrm{em}$ sensor at $\{t\}$ w.r.t. the sensor at $\{b\}$, an independent system for measuring the deflection of the continuum robot tip is obtained. This pose is 


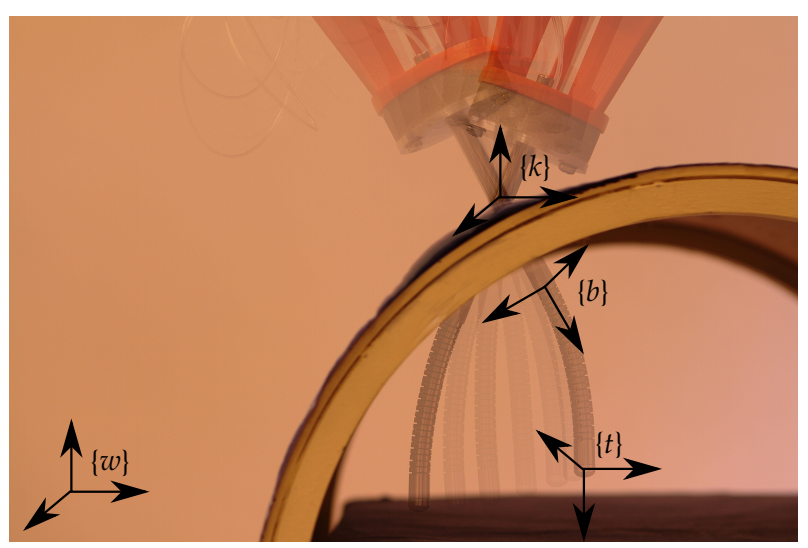

Fig. 6 The actual experimental set-up. The tip of the continuum robot is maintaining the orientation towards the ground, while travelling along a set-point trajectory. Simultaneously the rigid part of the robot is passing through the trocar.

then superimposed on the the pose of the rigid robot. Latter can obviously be measured by the different robot encoders. From this, the tip position measurement $y$ of the hybrid robotic system is obtained.

\subsection{Experimental parameters}

The tip position and orientation of the tool-tip frame $\{t\}$ were commanded to follow the pose of frame $\{f\}$, while this frame has been made to move so that it follows a simulated heartbeat movement. Such motion was programmed to take place along the $z$-axis of the object frame $\{o\}$. The incision point constraint is here represented by a stationary constraint by fixing the position of $\{k\}$ rigidly in space.

From spectral decomposition of the heart motion performed by Cavusoglu and Bebek [2], the first harmonic of heart motion is found at $2 \mathrm{~Hz}$ with frequency components going up to $26 \mathrm{~Hz}$. The results of this study indicate that the lower frequency components of heart motion typically result from breathing. Here a dominant mode can be found around approximately $0.37 \mathrm{~Hz}$. Overall, the power density of motion at frequencies higher than $8 \mathrm{~Hz}$ can be neglected. From these observations the following sinusoidal trajectory has been imposed on $\{f\}$ :

${ }_{o} \boldsymbol{T}^{f}=\left[\begin{array}{c|c}\boldsymbol{I}_{3 \times 3} & 0 \\ & A \sin (\omega t) \\ \hline \mathbf{0}_{1 \times 3} & 1\end{array}\right], \dot{x}_{u\{f\}}={ }_{o} \mathbf{t}^{f}=\left[\begin{array}{c}0 \\ 0 \\ A \omega \cos (\omega t) \\ \mathbf{0}_{3 \times 1}\end{array}\right]$

where $A$ is the amplitude of the sine wave (set to $10 \mathrm{~mm}$ ), and $\omega=2 \pi f$ is the angular frequency.
The position and orientation of the frames $\{k\}$ which defines the position of the trocar w.r.t. the position of the base of the rigid robot at frame $\{w\}$, and the position of the frame $\{o\}$ is summarised in table 2.

\begin{tabular}{c||c|ccc}
\hline \multirow{2}{*}{ frame } & orientation & \multicolumn{4}{c}{ position[mm] } \\
\hline$\{k\}$ & $\mathrm{x}$ & -650 & -50 & $\mathrm{y}$ \\
\hline \multirow{2}{*}{\begin{tabular}{c}
$\boldsymbol{I}_{3 \times 3}$ \\
\hline
\end{tabular}} & {$\left[\begin{array}{ccc}1 & 0 & 0 \\
0 & -1 & 0 \\
0 & 0 & -1\end{array}\right]$} & -670 & -50 & 80 \\
\hline
\end{tabular}

Table 2 Pose of the object frame $\{0\}$ and the trocar $\{k\}$ w.r.t. the world frame $\{w\}$ located at the base of the rigid robot.

Further on, the parameters of the controller used in the simulation and later in the experiments are given in table 3.

\begin{tabular}{|c|c|c|c|c|c|}
\hline $\operatorname{diag}\left(\boldsymbol{K}_{p}\right)$ & $\begin{array}{l}\text { tip pose } \\
\qquad \operatorname{diag}(W)\end{array}$ & $H$ & $\operatorname{diag}\left(\boldsymbol{K}_{p}\right)$ & $\begin{array}{l}\text { trocar } \\
\operatorname{diag}(\boldsymbol{W})\end{array}$ & $H$ \\
\hline 0.5 & 1 & 1 & 3 & 20 & 0.001 \\
\hline
\end{tabular}

Table 3 Controller parameters.

\subsection{Simulation}

First, the capability to track the above defined trajectory has been evaluated in a simulation scenario. Such simulation gives a first indication of the ability of the controller to cope with fast trajectory following, but in an ideal scenario, i.e. in the absence of modelling errors. Both the rigid robot and the continuum robot are here replaced by an idealized system, where the rigid robot accurately follows the imposed velocity and the kinematics of the continuum system is accurately described with the kinematic model given in ??.

Fig. 6 shows the results of the trajectory following simulation where the tip pose is plotted for the two different sine waves, with frequencies $f$ respectively at $0.37 \mathrm{~Hz}$ and $2 \mathrm{~Hz}$. An increase in tracking error can be observed. The error is present in both cases, however for the sine trajectory at $0.37 \mathrm{~Hz}$, the maximum errors remain below $0.1 \mathrm{~mm}$. For the more dynamic simulation at $2 \mathrm{~Hz}$ the error increases to $1 \mathrm{~mm}$.

This effect is expected to originate from the linearisation implicitly introduced by the Jacobian-based control, and in particular the way the tangent manifold (linearisation of forward kinematics around the current configuration) approximates the non-linear mapping 

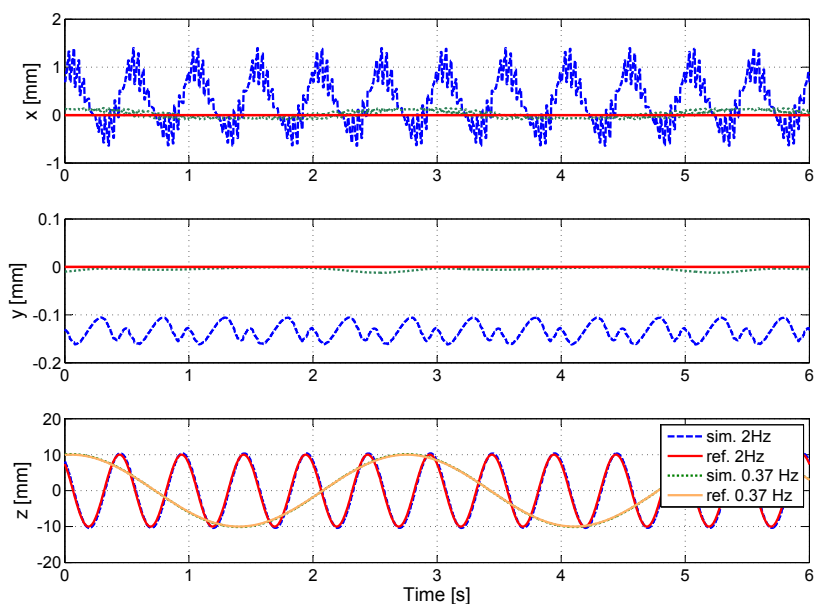

Fig. 7 Simulation results for two different values of reference trajectories at $0.37 \mathrm{~Hz}$ and $2 \mathrm{~Hz}$. The increase in the error for the $2 \mathrm{~Hz}$ reference is obvious, indicating a dependency of the tracking accuracy on the rate at which computations take place. Note that the graphs have different scale.

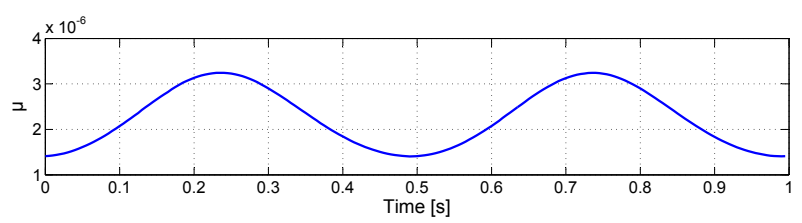

Fig. 8 The change of the manipulability index with the configuration of the robot in the simulation scenario.

between input $q$ and output $T$, in relation to the desired motion (in output space) within a single time step. The measures of Jacobian manipulability can be used here to assess the Jacobian properties. In particular by observing the singular values of the task Jacobian matrix $A$, where the largest singular value is (in average) 2.02 and the smallest $3.5 \cdot 10^{-3}$, it can be concluded how the Jacobian matrix $A$ is ill-conditioned. The overall manipulability index $\mu$ :

$\mu=\sqrt{\operatorname{det}\left(A A^{T}\right)}$,

is changing through different configurations of the robot. For the simulations performed for the case when $f=2 \mathrm{~Hz}$, the manipulability $\mu$ is shown on Fig. 7 .

The effect of low manipulability directly relates to the sampling time, and with lower dexterity of the robot higher sampling rates are required [17]. This effect is also visible in larger tracking errors when following faster trajectory in simulation (Fig. 6) even though the sample time is the same in both simulations. Furthermore, due to the non-linear nature of the robotic system, the amplitude and phase of these oscillations are highly related to the configuration of the robot itself and the position of the robot w.r.t. the location of the trocar and the location of $\{0\}$.
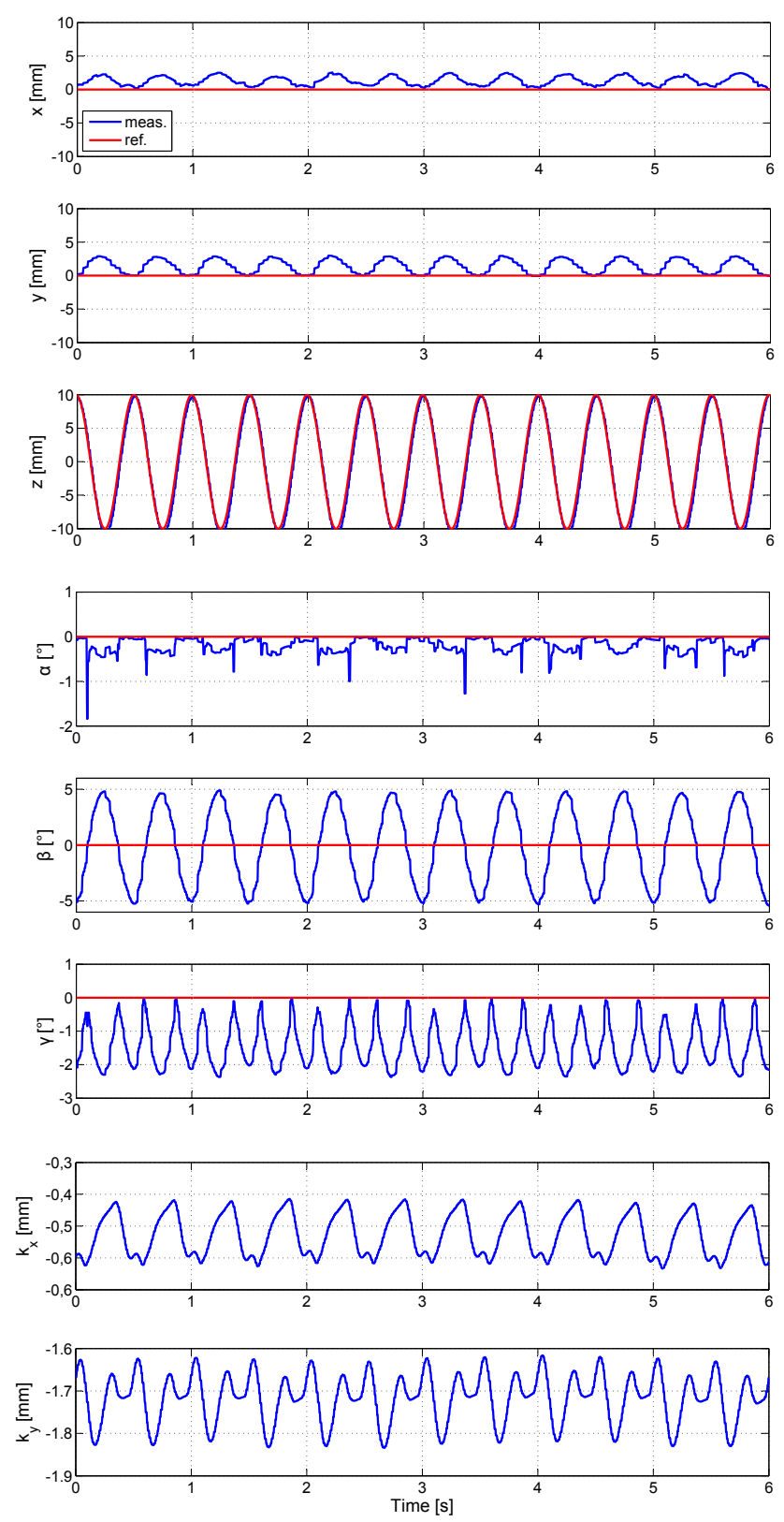

Fig. 9 Experimental results of sinusoidal trajectory following. The upper three graphs show the position of the tip. The middle three graphs show the orientation of the tip. The bottom two graphs indicate the position error of the trocar point in $x-y$ plane, expressed in $\{b\}$.

\subsection{Experiments}

Following the results obtained in simulation, an actual experiment was performed. This experiment corresponds to the simulated experiment in the sense that the position and the configuration of the rigid robot, the positions of the trocar point as well as the trajectories are identical.

Fig. 8 shows the results of a trajectory following experiment. Each graph shows the evolution of a single 
constraint that is set during the experiment. Overall the tracking performance is acceptable. For example this can be seen by observing the quality with which the sinusoidal reference trajectory is followed (third graph in the figure). However, even though the sinusoidal reference is applied only on the $z$ coordinate, some oscillations can also be observed in the other graphs showing that the system is not entirely decoupled. The position error remains however below $3 \mathrm{~mm}$ for both $x$ and $y$ coordinates. The orientation error goes up to $5^{\circ}$, whereas the error in maintaining a fixed trocar position remains below $2 \mathrm{~mm}$. The tracking errors during this experiment are thus larger than those observed in simulations. In particular a rise in tracking error of $1 \mathrm{~mm}$ for the $x$-component and $3 \mathrm{~mm}$ for the $y$-component was observed. The error in $z$ direction remains below $2 \mathrm{~mm}$.

The errors could originate from several sources: i) imperfect system assembly. Amongst others the alignment of muscles was not done as perfectly symmetric as assumed by the kinematics model. ii) Muscles have different $K$ coefficients. As such, each muscle has a different pressure to curvature ratio. iii) Some hysteresis appeared in the continuum robot response. iv) Dynamic effects that follow from the non-negligible mass of the robot, as well as the presence of non-modelled internal structural damping. $v$ ) Dynamics from the air pressure valves and the delay following from the relatively long air supply tubes. vi) The lower sampling rate of the $e m$ tracking system $(40 \mathrm{~Hz})$ as opposed to the $(100 \mathrm{~Hz})$ sampling rate of the rigid robot. . In further work the origin of this tracking error will be investigated more deeply. However the study of the continuum robot characterisation is beyond the scope of this paper.

\subsection{Discussion}

This subsection summarizes some additional observations that were made during the experiments and that can't be immediately observed from the experimental results alone.

Robot workspace It has been found that certain tool tip poses are not reachable with the proposed instrument configuration. In particular, the robot was unable to orient the tool accurately in the entire mock-up workspace. This follows from the fact that the continuum robot can only achieve a maximum bending angle within the range of $\pm 45^{\circ}$. Therefore, the ability of the robot to reach a desired pose depends largely on various parameters such as: the relative position of the incision point w.r.t. the rigid robot position, the configuration of the rigid robot and the proximity to its joint limits. These limitations can be overcome or mitigated by planning the procedure and calculating the optimal location of the rigid robot, or by devising new flexible instruments with larger range of angulation or with a certain amount of pre-curvature.

Sample time It has been observed in the simulation that the trajectory following accuracy improves with faster loop rates. At present, the loop rate is set to $0.01 \mathrm{~s}$ which is equal to the rate at which the joint velocity controller of the rigid robot works. Following the rationale given in Sec. 5.2 the employed hybrid-robotic mechanism requires faster loop rates due to the ill-conditioned robot Jacobian.

Hybrid robotic mechanism Another relevant observation resulting from this study relates to the use of the rigid robot employed in the scope of this work (KUKA LWR). The robot is a serial-chain robot with seven revolute joints. Since the proposed procedure requires insertion/retraction of the tool through the trocar and some pivoting about this point, the nature of the procedure could indicate that the employed rigid robotic mechanism is not the best possible option to achieve this task. We suggest that the rigid robot should be chosen in combination with the employed continuum robotic mechanism such that the more uniform manipulability of the overall hybrid robotic mechanism is achieved. A possible extension of this work could be to use the proposed simulation framework in order to identify and validate the best possible couple of rigid-continuum robot combinations for a given clinical application.

\section{Conclusion}

This paper proposes a hybrid robotic and control approach, combining a classical rigid robot with a continuum segment, in order to overcome the classical limitations of transapical heart surgery and, more generally, of keyhole surgery. The rigid robot allows 6DoF instrument positioning while the continuum robot adds 2 more DoF at the tip, effectively restoring the 2 DoFs that are lost when passing through a trocar. The continuum robot tip is compliant and as such introduces extra safety into the system, reducing the risk for tissue damage.

The paper shows how by using a control framework adapted from eTC, it becomes possible to control the tip degrees of freedom and make those follow a complex trajectory while respecting constraints imposed by the insertion point. The quality of the proposed approach is validated both in simulation and experiment. 
Although the proposed hybrid robotic system shows great potential for use in complex keyhole procedures, further works need to be conducted before allowing this system in a real clinical operating theatre. This includes a further study of the phenomena that explain the existence of a certain tracking error. Also the control framework must be evaluated in more detail to understand how the presence of conflicting constraints affect the overall outcome and whether any kind of performance guarantees can be made.

The effect of low manipulability and the existence of oscillations in the simulation due to the lower sampling times further stresses the importance for finding efficient modelling schemes for calculation of forward and differential kineamtics of continuum robots.

Acknowledgements Research funded by the European Commission's 7th Framework Programme FP7-ICT - Under grant agreement No 601021, EU project CASCADE.

\section{References}

1. E. Aertbeliën and J. De Schutter, eTaSL/etC: A constraintbased task specification language and robot controller using expression graphs, in IEEE/RSJ Proc. of International Conference on Intelligent Robots, 2014.

2. O. BEBEK AND M. CAvusoglu, Intelligent control algorithms for robotic-assisted beating heart surgery, Robotics, IEEE Transactions on, 23 (2007), pp. 468-480.

3. G. Borghesan, B. Willaert, T. De Laet, and J. De Schutter, Teleoperation in Presence of Uncertainties : a Constraint-Based Approach, in Proc. of 10th IFAC Symposium on Robot Control, 2012, pp. 385-392.

4. G. Chen, M. T. Pham, and T. Redarce, A guidance control strategy for semi-autonomous colonoscopy using a continuum robot, in Recent Progress in Robotics: Viable Robotic Service to Human, Springer, 2008, pp. 63-78.

5. A. Cheung and K. M. Lichtenstein, Illustrated techniques for transapical aortic valve implantation, Annals of Cardiothoracic Surgery, 1 (2012), pp. 231-239.

6. L. D. Daerden F., Pneumatic artificial muscles: actuators for robotics and automation, European Journal of Mechanical and Environmental Engineering, (2002), pp. 10-21.

7. J. De Schutter, T. De Laet, J. Rutgeerts, W. Decré, R. Smits, E. Aertbeliën, K. Claes, and H. Bruyninckx, Constraintbased Task Specification and Estimation for Sensor-Based Robot Systems in the Presence of Geometric Uncertainty, The International Journal of Robotics Research, 26 (2007), pp. 433-455.

8. W. Decré, R. Smits, H. Bruyninckx, and J. D. SChutTER, Extending itasc to support inequality constraints and noninstantaneous task specification., in IEEE Proc. of the Int. Conf. on Robotics and Automation, 2009.

9. A. Devreker, E. V. Poorten, A.Gijbels, P. T. Tran, H. D. Praetere, P. Herijgers, J. V. Sloten, and D. Reynaerts, Towards fluidic actuation for catheter-based interventions, in Proceedings Actuator, 2014.

10. I. Godage, E. Guglielmino, D. Branson, G. MedranoCERda, AND D. CALdwell, Novel modal approach for kinematics of multisection continuum arms, in Intelligent Robots and
Systems (IROS), 2011 IEEE/RSJ International Conference on, Sept 2011, pp. 1093-1098.

11. E. G. Hocking ANd N. M. Wereley, Analysis of nonlinear elastic behavior in miniature pneumatic artificial muscles, Smart Materials and Structures, 22 (2013), p. 014016.

12. I. S. INC, da vinci surgical system.

13. R. Kang, D. T. Branson, T. Zheng, E. Guglielmino, and D. G. CALDWELL, Design, modeling and control of a pneumatically actuated manipulator inspired by biological continuum structures, Bioinspiration \& Biomimetics, 8 (2013), p. 036008.

14. S. Kesner AND R. Howe, Position control of motion compensation cardiac catheters, Robotics, IEEE Transactions on, 27 (2011), pp. 1045-1055.

15. О. Кнатів, A unified approach for motion and force control of robot manipulators: The operational space formulation, IEEE Journal on Robotics and Automation, 3 (1987), pp. 43-53.

16. A. Lauten, A. Selle, M. Ferrari, A. Laube, W. Rademacher, A. Hamadanchi, B. Goebel, and H. Figulla, Rapid ventricular pacing during tavi frequently induces arrest and delayed recovery of microvascular perfusion, European Heart Journal, 34 (2014).

17. P. Leven and S. Hutchinson, Using manipulability to bias sampling during the construction of probabilistic roadmaps, in Robotics and Automation, 2002. Proceedings. ICRA '02. IEEE International Conference on, vol. 2, 2002, pp. 2134-2140 vol.2.

18. N. Mansard, O. Khatib, and A. Kheddar, A unified approach to integrate unilateral constraints in the stack of tasks, Robotics, IEEE Transactions on, 25 (2009), pp. 670-685.

19. M. T. Mason, Compliance and force control for computer controlled manipulators, Systems, Man and Cybernetics, IEEE Transactions on, 11 (1981), pp. 418-432.

20. R. M. Murray, Z. Li, And S. S. Sastry, A Mathematical Introduction to Robotic Manipulation, CRC Press, 1994.

21. J. Peirs, D. Reynaerts, H. Van Brussel, G. De Gersem, and H.-W. TANG, Design of an advanced tool guiding system for robotic surgery, in Robotics and Automation, 2003. Proceedings. ICRA '03. IEEE International Conference on, vol. 2, sept. 2003, pp. $2651-2656$ vol.2.

22. G. Robinson and J. B. C. Davies, Continuum robots - a state of the art, in Robotics and Automation, 1999. Proceedings. 1999 IEEE International Conference on, vol. 4, 1999, pp. 2849-2854 vol.4.

23. B. Rosa, C. Gruijthuijsen, B. Van Cleynenbreugel, J. Sloten, D. Reynaerts, and E. Poorten, Estimation of optimal pivot point for remote center of motion alignment in surgery, International Journal of Computer Assisted Radiology and Surgery, (2014), pp. 1-11.

24. P. Thienphrapa, A. Popovic, And R. TAylor, Guidance of a high dexterity robot under $3 d$ ultrasound for minimally invasive retrieval of foreign bodies from a beating heart, in Robotics and Automation (ICRA), 2014 IEEE International Conference on, May 2014, pp. 4869-4874.

25. B. Tondu And P. Lopez, Modeling and control of mckibben artificial muscle robot actuators, Control Systems, IEEE, 20 (2000), pp. 15-38.

26. D. Trivedi, A. Lotfi, AND C. RAhN, Geometrically exact models for soft robotic manipulators, Robotics, IEEE Transactions on, 24 (2008), pp. 773-780.

27. I. D. WALKER, Continuous backbone continuum robot manipulators, ISRN Robotics, vol. 2013 (2013), p. Article ID 726506.

28. R. J. Webster and B. A. Jones, Design and kinematic modeling of constant curvature continuum robots: A review, The International Journal of Robotics Research, (2010). 